\title{
Multifocus Image Fusion Based on Multiresolution Transform and Particle Swarm Optimization
}

\author{
Yushu Liu, Mingyan Jiang \\ School of Information Science and Engineering, \\ Shandong University, \\ Jinan, 250100, China \\ E-mail: ysliu.82@gmail.com, \\ corresponding author: jiangmingyan@sdu.edu.cn
}

\author{
Chuanzhu Liao \\ Department of Electronic Engineering, \\ Zhangzhou Institute of Technology, \\ Zhangzhou, 363000, China \\ E-mail: zzylcz@126.com
}

\begin{abstract}
In order to get an image with every object in focus, an image fusion process is required to fuse the images under different focal settings. In this paper, a novel multifocus image fusion algorithm based on multiresolution transform and particle swarm optimization (PSO) is proposed. Firstly the source images are decomposed into lowpass subbands coefficients and highpass subbands coefficients by the nonsubsampled contourlet transform (NSCT). Then, different fusion rules are applied for low and high frequency NSCT coefficients. Finally the fused image is reconstructed by the inverse NSCT transform. The experiment results demonstrate that the proposed method is effective and can provide better performance than the method based on the wavelet transform and the nonsubsampled contourlet transform.
\end{abstract}

Keywords- nonsubsampled contourlet transform; particle swarm optimization; image fusion

\section{INTRODUCTION}

Image fusion has become an important part of image processing. Multiple images can be taken from two or more sensors in the same time or different times for a specific image or scene. Image fusion is the process of combining the relevant information of these images to obtain a new explanation of the image or scene. The purpose of image fusion is reducing uncertainty. The fused image can better describe the scene than any of the individual source images. Image fusion has already applied in many fields, such as digital imaging, medical imaging, remote sensing, and machine vision [1-3].

When scene contains objects of different depth of focus, it is impossible to display all areas of the picture very well. Therefore, if one object in the scene is in focus, the other objects at different distances from the camera will be out of focus and blurred. So in order to get all the objects focused in one image multifocus image fusion is needed. Several images of a scene are taken with focus on different parts. Then these images are fused with the purpose that the objects will be focused in the resulting image [4-5].

Many algorithms have been developed to resolve the image fusion problem. In those methods, the fusion schemes based on Multiscale transform have attracted quite a lot of research attention, such as wavelet [2], contourlet [6], and nonsubsampled contourlet transform [7].

In this paper, a nonsubsampled contourlet transform (NSCT) based fusion algorithm with particle swarm optimization (PSO) is proposed. The source images are decomposed using nonsubsampled contourlet transform. Depending on the nature of the transformed coefficients, they are fused by different fusion rules. Then the fused image is obtained by applying inverse nonsubsampled contourlet transform on the fused coefficients.

The rest of the paper is organized as follows: section II reviews the basic theory of nonsubsampled contourlet transform and particle swarm optimization, and also presents the procedure of the proposed fusion algorithm. Section III shows the experiment results and its comparison with the other methods. Finally conclusions are given in section IV.

\section{METHOD}

\section{A. Nonsubsampled Contourlet Transform}

NSCT is a kind of shift invariant, multiscale, multidirectional transform [8]. In contourlet transform the cause of shift variance is due to the presence of downsampler and upsampler in both the laplacian pyramid (LP) and the directional filter bank (DFB). To reduce it, nonsubsampled pyramid structure and nonsubsampled directional filter bank are employed in NSCT. NSCT provides a better frequency selectivity and regularity than contourlet transform. Fig.1 shows the structure of NSCT. The NSCT is constructed by combining the nonsubsampled pyramid filter banks (NSPFB) and nonsubsampled directional filter banks (NSDFB) steps. The NSPFB gives the multiscale property while directionality is given by the NSDFB. The input image is decomposed in lowpass subband and highpass subband (Fig.1 (a)). Then NSDFB is applied on the highpass subband to obtain directional subbands (Fig.1 (b)). The lowpass subband is further decomposed by the NSPFB until up to the desired level of decomposition.

\section{B. Particle Swarm Optimization}

Particle swarm optimization (PSO), a population-based optimization algorithm, is inspired by the social behavior of animals such as fish schooling and bird flocking [9, 10]. In 
PSO, each particle represents a potential solution to a complex problem in the search space. Each particle has two attribute values: fitness determined by the problem and velocity to decide the flying. Each particle can learn from the previous experiences of all particles. That is, the position of a particle is influenced by the best position of its own experience and the best particle position in the swarm. The fitness function is used to measure the performance of each particle.

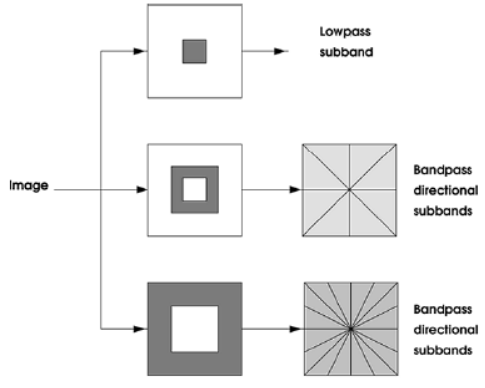

(a)

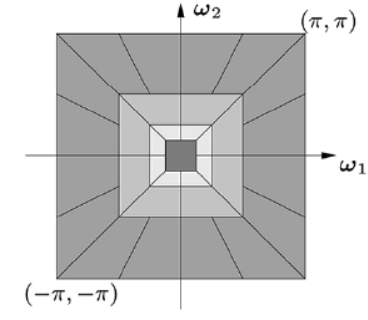

(b)
Fig. 1. Nonsubsampled contourlet transform. (a) Decomposition framework of NSCT. (b) Idealized frequency partitioning obtained with the NSCT

During the search process, the new velocity of particle $i$ at dimension $d$, i.e., $v_{i}^{d}$, and the new position of particle $i$ at dimension $d$, i.e., $x_{i}^{d}$, are updated, respectively, by

$$
\begin{gathered}
v_{i}^{d}(t+1)=\omega \cdot v_{i}^{d}(t)+c_{1} \cdot r_{1}(t) \cdot\left(p_{i}^{d}(t)-x_{i}^{d}(t)\right) \\
+c_{2} \cdot r_{2}(t) \cdot\left(p_{g}^{d}(t)-x_{i}^{d}(t)\right) \\
x_{i}^{d}(t+1)=x_{i}^{d}(t)+v_{i}^{d}(t+1)
\end{gathered}
$$

where $t$ denote the iteration counter, $\omega$ is the inertia weight controlling the impact of the previous velocity, $c_{1}$ and $c_{2}$ are learning constants, $r_{1}$ and $r_{2}$ are random variables in the range $[0,1], p_{i}$ is the best position of particle $i$, and $p_{g}$ is the best position of all particles within iteration $t$. follows.

The processing steps of PSO are briefly described as

Step 1: Initialize the population. Each particle has its own random velocity and position.

Step 2: Determine the fitness function value of each particle.

Step 3: Find the best position of its own experience of each particle.

Step 4: Find the position of the best particle.

Step 5: Update the velocity and position of each particle via equation (1) and (2).

Step 6: Check if the current optimization solution can be accepted or the stopping criterion is satisfied. Otherwise, go to Step 2.

PSO, as an evolutionary computing algorithm, is suitable for solving a complex problem with approximate solutions and it only requires primitive mathematical operations, which means that PSO can be implemented easily.

\section{The proposed image fusion method}

In this paper, image decomposition is performed by the NSCT. The NSCT not only retains the property of contourlet, but also has a very important characteristic of shiftinvariance. When it is applied in image fusion, the size of different subbands is same, so it is easy to find the relationship between different subbands, which is good for designing the fusion rule and helpful for avoiding the pseudo-Gibbs phenomenon. Therefore, NSCT is suitable for image fusion.

In order to improve the quality of the fused image, two different focus measurements are presented. The whole process is divided in three steps: Decomposition of source images in NSCT, Fusion of NSCT coefficients of source images and reconstruction from fused NSCT coefficients. After transform the source images into NSCT domain, we can get the coefficients of both source images as,

$$
\begin{aligned}
& f_{A}(x, y) \rightarrow\left(C_{1}^{A}, C_{2}^{A}, \cdots, C_{j-1}^{A}, C_{j}^{A}, B_{j}^{A}\right) \\
& f_{B}(x, y) \rightarrow\left(C_{1}^{B}, C_{2}^{B}, \cdots, C_{j-1}^{B}, C_{j}^{B}, B_{j}^{B}\right)
\end{aligned}
$$

where $f_{A}$ and $f_{B}$ are the source images, $B_{j}$ are the lowfrequency coefficients and $C_{j}$ are the high-frequency coefficients at scale $j$.Then we fuse the coefficients of the source images. Because the low and high frequency coefficients have different properties, different fusion rules are applied on them. Considering the approximate information of the image is constructed by the low-frequency coefficients, hence they are fused by taking average rule. Suppose that $B^{F}(x, y)$ is the fused low-frequency coefficients, then

$$
B^{F}(x, y)=\frac{B^{A}(x, y)+B^{B}(x, y)}{2}
$$

where $B^{A}(x, y)$ and $B^{B}(x, y)$ are the low-frequency coefficients of image $A$ and image $B$ respectively at pixel location $(x, y)$.

The high-frequency coefficients contain important information of the images such as edges, texture features. The weight averaging is applied to them:

$$
C_{j}^{F}(x, y)=\alpha \cdot C_{j}^{A}(x, y)+(1-\alpha) \cdot C_{j}^{B}(x, y)
$$

where $\alpha$ and $1-\alpha$ are scalar weights, $C_{j}^{A}(x, y)$ and $C_{j}^{B}(x, y)$ are the high-frequency coefficients of image $A$ and image $B$ respectively at scale $j$ and pixel location $(x, y)$, and $C_{j}^{F}(x, y)$ is the fused high-frequency coefficients at scale $j$ and pixel location $(x, y)$. In this paper, we use 
PSO to determine the parameter $\alpha$. For different location of each subband in different direction the value of parameter $\alpha$ can be obtained by PSO, so we can get the proper fused coefficients.

After applying fusion rules on NSCT coefficients, we have the coefficients of the fused image, on which we apply inverse NSCT to obtain the final fused image.

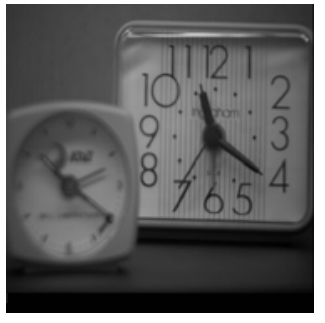

(a) Image A

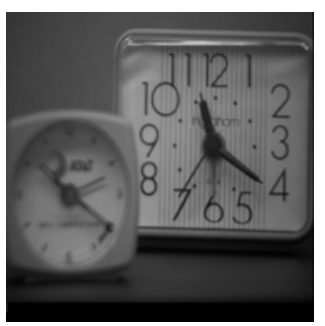

(c) DWT based fusion

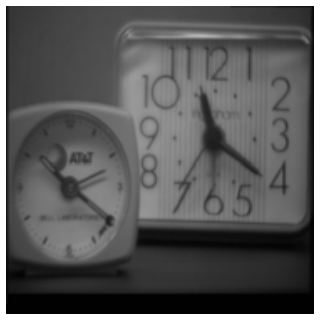

(e) PSO based fusion Fig. 2. Fusion results for multifocus images.

TABLE I. COMPARION OF FUSION RESULTS FOR MULTIFOCUS IMAGES

\begin{tabular}{|c|c|c|}
\hline & MI & $Q^{A B / F}$ \\
\hline DWT based fusion & 6.3419 & 0.6151 \\
\hline NSCT based fusion & 6.6761 & 0.6671 \\
\hline PSO based fusion & 7.0399 & 0.5567 \\
\hline Proposed method & 6.7156 & 0.6764 \\
\hline
\end{tabular}

\section{EXPERIMENTAL RESULTS AND ANALYSIS}

This section gives the visual and quantitative evaluation of the proposed method and its comparison with discrete wavelet transform (DWT), nonsubsampled contourlet transforms (NSCT) and particle swarm optimization (PSO) based fusion method.
The presented method is tested with multifocus images of size $512 \times 512$. Fig.2 (a) and Fig.2 (b) show a pair of tested images containing two clocks with different distances toward the camera and only one clock in either image is in focus. For NSCT, five scales of decomposition are used. The NSPFB is '9-7' and the NSDFB is 'pkva'. The PSO parameters are set as following: the population size, two learning constants $c_{1}$ and $c_{2}$ are empirically set to 30, 2 and 2 respectively; inertial factor $\omega$ linearly decreases from 0.9 to 0.4 ; the iteration times is 100 . The experiment result of proposed method is shown in Fig. 2. The visual results of the other three methods are also shown in Fig. 2. From these results it is clear that our method gives better results than the other three methods.

The visual analysis is not the only performance evaluation criterion because sometimes it does not give the clear image. Hence some quantitative measurements are also been used. We use mutual information (MI) [11] and $Q^{A B / F}$ [12] for this purpose. MI calculates how much information of the source images is transferred to the fusion image. $Q^{A B / F}$ uses the Sobel edge detection operator to measure how much information of the source images removed to the fusion result. If the values of $\mathrm{MI}$ and $Q^{A B / F}$ are higher, it means that the fusion performance of the method is better. Values of these quantitative measures of different methods for the tested images are given in table I . As we know that NSCT is the shift invariance version of CT, and it has more directional subbands than DWT. The influence of this can be observed by viewing the improvements of results. Our method gets better result than using NSCT or PSO to fuse individually. Analyzing the table, we can observe that the proposed method has the highest value of $Q^{A B / F}$ than other three methods. The value of $\mathrm{MI}$ is a little lower than PSO but higher than other two methods. Generally speaking our method obtains an acceptable and better result. Hence combining both the qualitative and quantitative analysis we can say that the performance of the propose method is better than other methods.

\section{CONCLUSIONS}

In this paper, a new method of multifocus image fusion, which combines the NSCT and PSO, is presented. Nonsubsampled contourlet transform is an extension of contourlet transform with shift invariance characteristic. We have used two different fusion rules for low pass and high pass NSCT coefficients. Average rule is adopted in the lowfrequency coefficients. PSO is used to obtain the proper fusion weight parameter of the high-frequency coefficients. Multifocus images are used to evaluate the performance of the proposed algorithm qualitatively as well as evaluated with two quality metrics (mutual information and $Q^{A B / F}$ ). Experiment results shows that the proposed method outperforms the wavelet, NSCT and PSO based methods.

\section{ACKNOWLEDGMENT}


This work is supported by the National Natural Science Foundation of Shandong Province of China under Grant No. ZR2010FM040 and Special Funding Project for Independent Innovation Achievements transform of Shandong Province under Grant No. 2009ZHZX1A0108, No.2010ZHZX1A1001

\section{REFERENCES}

[1] Z.Zhang and R.S. Blum, "A categorization of multiscaledecomposition-based image fusion schemes with a performance study for a digital camera application,” Proc. IEEE, vol. 87, no. 8, pp. 13151326, Aug. 1999.

[2] G. Pajares and J. Cruz, "A wavelet-based image fusion tutorial," Pattern Recognition, vol. 37, no. 9, pp. 1855-1872, sep. 2004.

[3] A.A. Goshtasby and S. Nikolov, "Image fusion: Advances in the state of the art,” Information Fusion, vol. 8, no. 2. pp. 114-118, Apr. 2007.

[4] G. Piella, "A general framework for multiresolution image fusion: From pixels to regions," Information Fusion, vol. 4, no. 4, pp. 259280, Dec. 2003.

[5] S.T. Li, J.T. Kwok, I.W. Tsang, and Y.N. Wang, "Fusing images with different focuses using support vector machines,” IEEE Trans. Neural Network, vol. 15, no. 6, pp. 1555-1561, Nov. 2004.
[6] L.Yang, B.L. Guo and W. Ni, "Multimodality medical image fusion based on multiscale geometric analysis of contourlet transform," Neurocomputing, vol.72, no. 26, pp. 203-211, Dec. 2008.

[7] Q. Zhang and B.L. Guo, "Multi-focus image fusion using the nonsubsampled contourlet transform," Signal Process, vol. 89, no. 7, pp.1334-1346, Jul. 2009.

[8] A.L. da Cunha, J.P. Zhou, and M.N. Do, "The Nonsubsampled Contourlet Transform: Theory, Design, and Applications," IEEE Trans. Image Processing, vol.15, no.10, pp.3089-3101, Oct. 2006.

[9] J. Kennedy and R. Eberhart, "Particla swarm optimization," Neural Networks, Proceedings., IEEE international conference on, vol. 4, pp. 1942-1948, Nov/Dec 1995.

[10] R. Eberhart and J. Kennedy, "A new optimizer using particle swarm theory," Proc. of the Sixth International Symposium on Micro Machine and Human Science, pp. 39-43, Oct 1995.

[11] G.H. Qu, D.L. Zhang and P.F. Yan, "Information measure for performance of image fusion,” Electronic Letters, vol. 38, no. 7, pp. 313-315, 2002.

[12] V. Petrovic and C. Xydeas.H, "On the effects of sensor noise in pixellevel image fusion performance," Proc. of the Third International Conference on Image fusion, vol. 2, pp. 14-19, July 2009 